\title{
Supporting Information: Amylation of Toluene by Solid Acid Catalysis
}

\author{
Michele Cowley, Arno de Klerk * and Reinier J. J. Nel \\ Fischer-Tropsch Refinery Catalysis, Sasol Technology Research and Development, \\ P.O. Box 1, Sasolburg 1947, South Africa. \\ Tel: +27 16 960-2549, Fax: +27 11 522-3517, E-mail: arno.deklerk@sasol.com
}

\section{Fischer-Tropsch feedstock}

Background. During high temperature Fischer-Tropsch (HTFT) synthesis as practised commercially in South Africa by Sasol in Secunda and PetroSA in Mossel Bay, the main product for each carbon number is the linear $\alpha$-olefin. ${ }^{1}$ Since the products follow a SchultzFlory distribution, odd-numbered and even-numbered $\alpha$-olefins are produced, unlike $\alpha$-olefin synthesis from ethene which produces only even-numbered $\alpha$-olefins. HTFT produces mostly light material and 1-pentene is consequently one of the most abundant species in the Fischer-Tropsch product.

The 1-pentene is purified from the Fischer-Tropsch product by distillation. However, 2-methyl-1-butene (2M1B) is close-boiling to 1-pentene and etherification with methanol is used to remove the 2M1B before purification of the 1-pentene. This is done in campaign operation on one of the 1-hexene purification trains at the Synfuels refinery in Secunda. This is possible because similar technology is used for 1-pentene and 1-hexene purification. ${ }^{2}$

Detail analysis of 1-pentene feed. The 1-pentene feed was analysed by gas chromatography with flame ionisation detector (GC-FID). Identification was done by gas chromatography with mass spectrometer (GC-MS) and by making use of model compounds (Table S1). Care was taken to check for the presence of dienes and oxygenates and none could be found.

\section{Amyl toluene synthesis over solid phosphoric acid}

Background. Solid phosphoric acid (SPA) was used to see what products could be expected from acid catalysed alkylation of toluene with 1-pentene at elevated temperature and pressure. 
Experimental. Toluene (92.4 g, Riedel-De Haën \#32249, 99.7\%), SPA (2.0 g, C84-3-01 AFCAT) and dodecane (2.8 g, as internal standard, Aldrich, 45,711-6, +99\%) were loaded in a $200 \mathrm{ml}$ autoclave and sealed. The reactor was leak tested with nitrogen. The nitrogen was released and the pressure adjusted to $1 \mathrm{MPa}$. The reactor was heated to $180^{\circ} \mathrm{C}(60 \mathrm{~min})$. On reaching $180^{\circ} \mathrm{C}, 1$-pentene $(7.0 \mathrm{~g}$, Table $\mathrm{S} 1)$ was introduced into the reaction via a steel bomb and the pressure adjusted with nitrogen to 3.8 MPa. Samples were taken at regular intervals with a double lock system that allows for the cooling of the sample under pressure. The reaction was stirred at $700 \mathrm{rpm}$. The samples were analysed with GC-FID (Agilent Technologies $6890 \mathrm{~N}$ ). A Pona $50 \mathrm{~m} \times 200 \mu \mathrm{m} \times 0.5 \mu \mathrm{m}$ Methyl Siloxane column (Agilent \#190915-001) was used with a 100:1 split ratio. The temperature programme started at $40^{\circ} \mathrm{C}$ for 10 minutes, followed by a $4^{\circ} \mathrm{C}$ per minute ramp to $250^{\circ} \mathrm{C}$ and a holding time of 10 minutes at $250^{\circ} \mathrm{C}$.

Results and discussion. There were mainly eight mono-alkylated amyltoluene species (Figure S1), although some other species were detected in much lower concentration. The rate of 1-pentene double bond isomerisation is much faster than the rate of skeletal isomerisation. It was therefore expected that the main products would be the ortho-, metaand para-isomers of 1-metylbutyltoluene and 1-ethylpropyltoluene. 2-Methyl-2-butene and 2-methyl-1-butene will yield the same product, 1,1-dimethylpropyltoluene. Only the metaand para-isomers were expected, as literature indicated that when a tert-alkyl is used, no ortho-product is formed. ${ }^{3}$

${ }^{13} \mathrm{C}-\mathrm{NMR}$ simulations showed that it should be possible to differentiate between the different isomers in a mixture without purification of the individual compounds. $\mathrm{A}^{13} \mathrm{C}-\mathrm{NMR}$ of the mixture showed the presence of nine isomers when looking at the ring carbon connected to the amyl group $(140-150 \mathrm{ppm})$. At this point the small peak (Figure 1 at 37 minutes) is assigned to the ortho-isomer of 1,1-dimethylpropyltoluene, which was not expected to be formed.

\section{Grignard synthesis of 1-ethylpropyltoluene isomers}

Background. The identification of ortho-, meta- and para-isomers of amyltoluene cannot easily be done by mass spectrometry, since the breakdown patterns are very similar. The 
synthesis of the pure compounds is the surest way to identify all the isomers, but is time consuming and costly. Grignard syntheses of only the 1-ethylpropyltoluene isomers were therefore done.

Experimental. The method was taken from literature. ${ }^{4,5}$ Dry ethoxyethane $(30 \mathrm{ml}$, Aldrich \#47,249-2, +98\%) was placed in a round bottom flask and magnesium (5.9 g, Aldrich \#20,090-5, 98\%) added, after its oxide layer was removed. The bromotoluene (50 g: 2bromotoluene, Aldrich \#B8,200-6, 99\%; 3-bromotoluene, Aldrich \#16,721-5, 98\%; 4bromotoluene, Aldrich \#B8,220-6, 98\%) and $50 \mathrm{ml}$ ether mixture was drop-wise added over a period of 1 hour and then allowed to reflux for 2 hours. The solution was allowed to cool and a further $30 \mathrm{ml}$ of the ether was added. 3-Pentanone (25.2 g, Aldrich \#34,512-1, +99\%) was drop-wise added carefully controlling the reaction rate. Once the addition was completed, the reaction mixture was refluxed for 2 hours and then cooled to room temperature before adding $50 \mathrm{ml}$ strong acid. (Initially sulphuric acid was used, but it was found that para-toluene sulfonic acid was required to completely convert the alcohol to the olefin). The product was purified by distillation and hydrogenated at $3 \mathrm{MPa}$ with a Pd/C catalyst (Aldrich \#20,569-9). The products were analysed by GC-MS and NMR.

Results and discussion. The syntheses were confirmed by NMR analysis and subsequently used to identify the isomers made during $\mathrm{AlCl}_{3}$ Friedel-Crafts alkylation.

\section{$\mathrm{AlCl}_{3}$ Friedel-Crafts alkylation of toluene with 1-pentene and 3-methyl-1-butene}

Background. Friedel-Crafts alkylation with $\mathrm{AlCl}_{3}$ is not skeletally isomerising. Alkylation with 1-pentene would therefore yield 1-methylbutyltoluene (1) and 1-ethylpropyltoluene (2). Since the 1-ethylpropyltoluenes were already synthesised, it would be possible to identify the 1-methylbutyltoluenes in the reaction product. Likewise alkylation with 3-methyl-1-butene would yield 1,1-dimethylpropyltoluene (3) and 1,2-dimethylpropyltoluene (4).

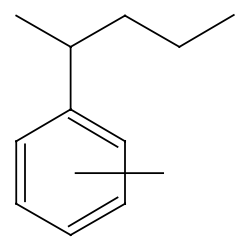

(1)

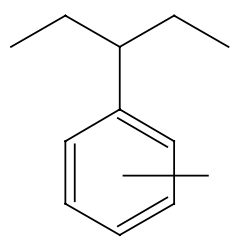

(2)

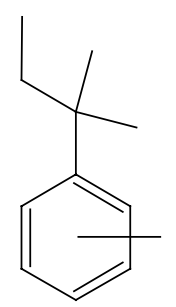

(3)

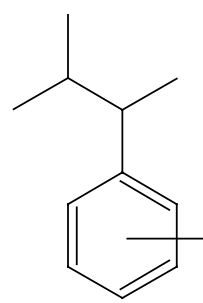

(4) 
Experimental. The equipment was dried using a stream of nitrogen and a heatgun. $\mathrm{AlCl}_{3}$ (21.0 g, Alfa Aesar \#12298) and toluene (39.4 g, Aldrich \#17,996-5, 99\%) were placed in the round bottomed flask and stirred at $0^{\circ} \mathrm{C}$. The 1-pentene $(33.4 \mathrm{~g}$, Table $\mathrm{S} 1)$ was placed in a pressure equalizing funnel and added drop-wise over a period of 30 minutes. After the addition was completed, the reaction mixture stirred at room temperature for 2 hours. Water (300 ml) was added twice and separated in a separating funnel. The product was analysed by GC-MS and GC-FID. A Pona $50 \mathrm{~m} \times 200 \mu \mathrm{m} \times 0.5 \mu \mathrm{m}$ Methyl Siloxane column (Agilent \#190915-001) was used with a 100:1 split ratio. The temperature programme was different to that used for the SPA work: $40^{\circ} \mathrm{C}$ for 5 minute, ramping from $40^{\circ} \mathrm{C}$ to $120^{\circ} \mathrm{C}$ at $4^{\circ} \mathrm{C}$ per minute and from $120^{\circ} \mathrm{C}$ to $300^{\circ} \mathrm{C}$ at $20^{\circ} \mathrm{C}$ per minute and then holding it for 6 minutes at $300^{\circ} \mathrm{C}$. The same procedure was used for alkylation with 3-methyl-1-butene (Sasol, 99.4\% by analysis).

Results and discussion. The reaction with 1-pentene yielded mono- to di-alkylated products in a ratio of 1:0.86. Six amyltoluene isomers could be separated chromatographically (Table S2 and Figure S2a). The electron impact fragmentation sequence for 1-methylbutyltoluene and 1-ethylpropyltoluene were expected to be different and it was used to interpret the mass spectra (Figures S2b-c and S3). This allowed identification of these species by GC-MS. The differentiation between the 1-ethylpropyltoluene isomers could be made by spiking the reaction mixture with the pure compounds prepared by Grignard synthesis. The 1methylbutyltoluene isomers were identified by analogy.

Four isoamyltoluene isomers could be separated from the alkylation with 3-methyl-1butene (Table S3 and Figure S4a), which yielded mono-, di- and tri-alkylated products in the ratio 1:0.36:0.18. The fragmentation sequence for 1,1-dimethylpropyltoluene and 1,2dimethylpropyltoluene were expected to be different and it was used to interpret the mass spectra (Figures S4b-c and S5). The differentiation between the meta- and para-isomers was based on the relative amount of the two isomers, the para-isomer is assumed to be formed preferentially, as with 1-pentene.

It was difficult to distinguish between the isomers in the reaction product when skeletal isomerisation occurred. The retention times of 1-methylbutyltoluene and 1ethylpropyltoluene overlaped with that of 1,1-dimethylpropyltoluene and 1,2- 
dimethylpropyltoluene. It was therefore not possible to unambiguously separate the amyltoluene and isoamyltoluene species as reported in the accompanying paper.

\section{Synthesis and identification of diamyl and decyl toluene species}

Background. Alkylation and oligomerisation are both acid catalysed reactions of olefins that occurred in parallel. In the present investigation $\mathrm{C}_{10}$-alkyltoluene could therefore be either due to dialkylation with pentene, or alkylation with a $\mathrm{C}_{10}$-olefin (product of pentene dimerisation). The latter was less likely to form, but this had to be verified.

Experimental. The same procedure as for the $\mathrm{AlCl}_{3}$ Friedel-Crafts alkylation of 1-pentene was used. To synthesis the decyltoluene, 1-decene (Aldrich \#D180-7, 94\%) was used as starting material.

Results and discussion. The chromatograms of the diamyltoluenes and decyltoluenes (Figure S6) show that there is some overlap. It should also be noted that the decyltoluenes that were synthesised, were not skeletally branched, apart from some branched impurities (about $3-4 \%$ ). It is therefore possible that the overlap could be even more, but not expected, because the branched alkyl aromatics have similar retention times.

\section{Acknowledgements}

All work was performed at Sasol Technology Research and Development and permission to publish the results is appreciated. Most of the amyltoluene syntheses and identification work were done by one of the authors (RJJN) and reported internally as FTRC 0641 (SPA) and FTRC 0691 ( $\mathrm{AlCl}_{3}$ Friedel-Crafts). The Grignard syntheses were done by Bradley Miller and RJJN and reported internally as FTRC 0661.

\section{Literature cited}

(1) Steynberg, A. P.; Dry, M. E. (eds) Fischer-Tropsch technology; Elsevier: Amsterdam, 2004.

(2) De Klerk, A. Etherification of $\mathrm{C}_{6}$ Fischer-Tropsch material for linear $\alpha$-olefin recovery. Ind. Eng. Chem. Res. 2004, 43, 6349. 
(3) Schlatter, M. J.; Clark, R. D. t-Alkyl groups. I. Orientation of $t$-alkylation products of toluene and ethylbenzene. J. Am. Chem. Soc. 1953, 75, 361.

(4) Furniss B. S.; Hannaford, A. J.; Smith P. W. G.; Tatchell, A. R. Vogel's Textbook of Practical Organic Chemistry (5ed); Longman: New York, 1989, 826-832.

(5) Roberts, R. M.; Gilbert, J. C.; Rodewald, L. B.; Wingrove, A. S. Modern Experimental Organic Chemistry (4ed); Holt-Saunders: Philadelphia, 1985, 545-558. 
Table S1. Detailed analysis of 1-pentene used for the experimental work.

\begin{tabular}{ll}
\hline Compound & Mass \% \\
\hline $\mathrm{C}_{4}$ - hydrocarbons & $<0.1$ \\
2-methylbutane (isopentane) & 0.7 \\
1-pentene & 99.0 \\
2-methyl-1-butene & 0.2 \\
$\mathrm{C}_{5}+$ hydrocarbons & 0.1
\end{tabular}

Table S2. Amyltoluene selectivity during $\mathrm{AlCl}_{3}$ alkylation of toluene with 1-pentene

\begin{tabular}{ll}
\hline Amyltoluene isomer & Mass \% \\
\hline$o$-1-methylbutyltoluene & 5.0 \\
$m$-1-methylbutyltoluene & 29.8 \\
$p$-1-methylbutyltoluene & 36.0 \\
$o$-1-ethylpropyltoluene & 1.5 \\
$m$-1-ethylpropyltoluene & 11.6 \\
$p$-1-ethylpropyltoluene & 16.1
\end{tabular}

Table S3. Isoamyltoluene selectivity during $\mathrm{AlCl}_{3}$ alkylation of toluene with 3-methyl-1butene

\begin{tabular}{ll}
\hline Isoamyltoluene isomer & Mass \% \\
\hline$m$-1,1-dimethylpropyltoluene & 20.7 \\
$p$-1,1-dimethylpropyltoluene & 50.5 \\
$m$-1,2-dimethylpropyltoluene & 12.6 \\
$p$-1,2-dimethylpropyltoluene & 16.2
\end{tabular}


Figure S1. Chromatogram showing amyltoluene region after alkylation over SPA.

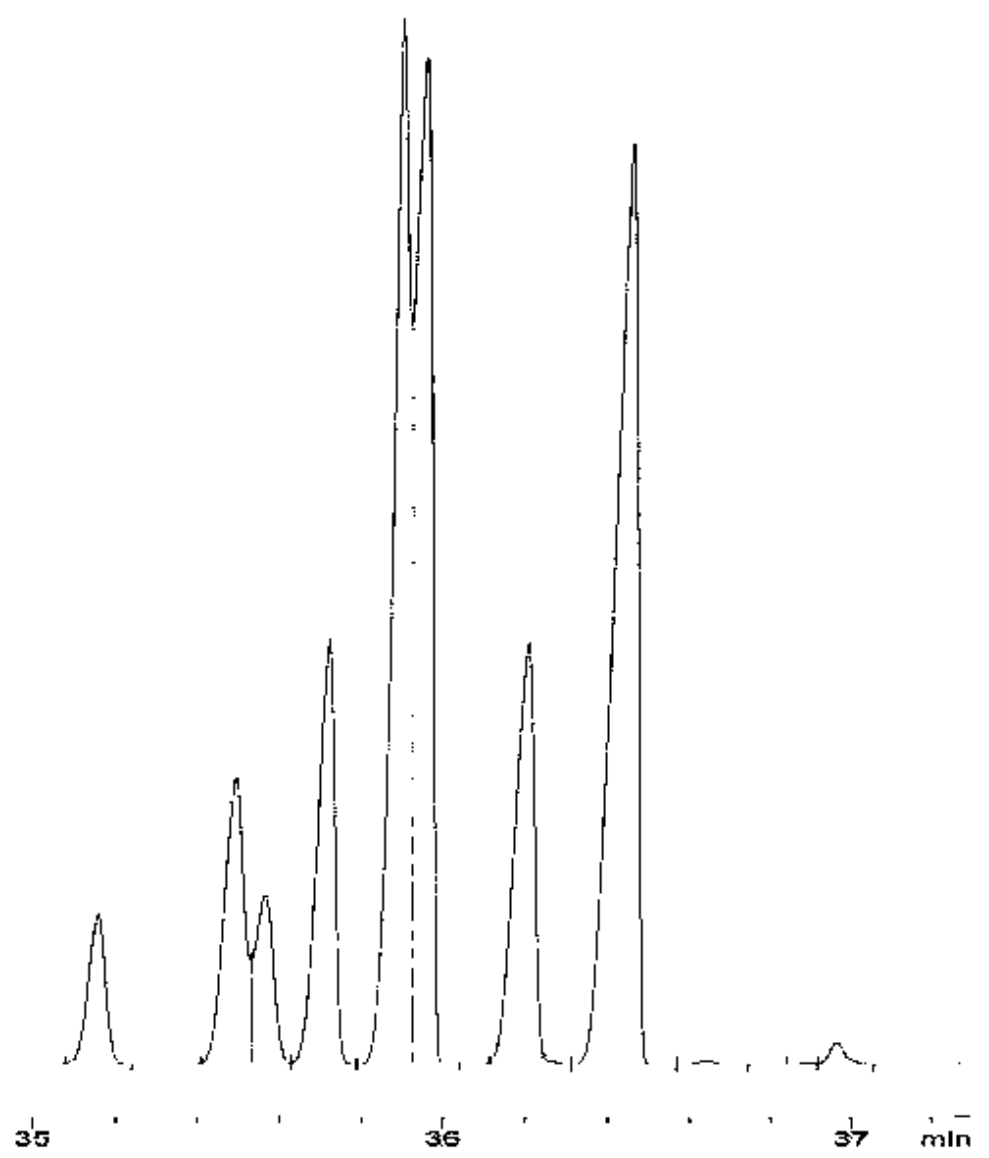


Figure S2a. Chromatogram of $\mathrm{AlCl}_{3}$ catalysed alkylation of toluene with 1-pentene showing: (a) $m$-1-ethylpropyltoluene, (b) $p$-1-ethylpropyltoluene, (c) $o$-1-ethylpropyltoluene, (d) $m$-1methylbutyltoluene, (e) $p$-1-methylbutyltoluene and (f) $o$-1-methylbutyltoluene.

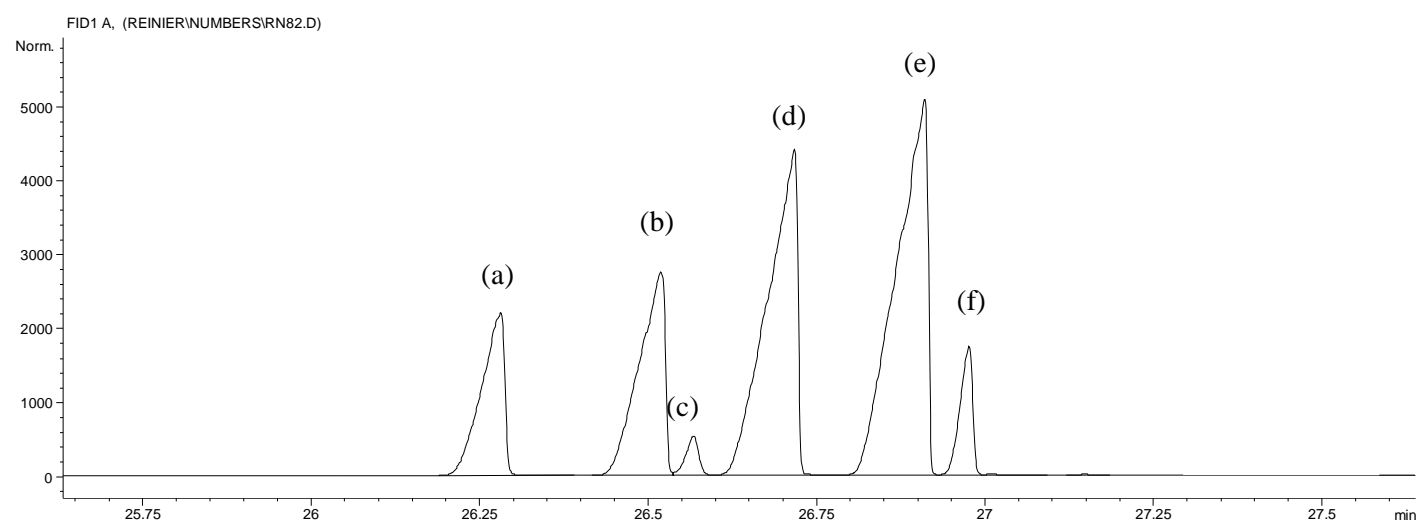

Figure S2b. Electron impact mass spectrum of (a) m-1-ethylpropyltoluene.

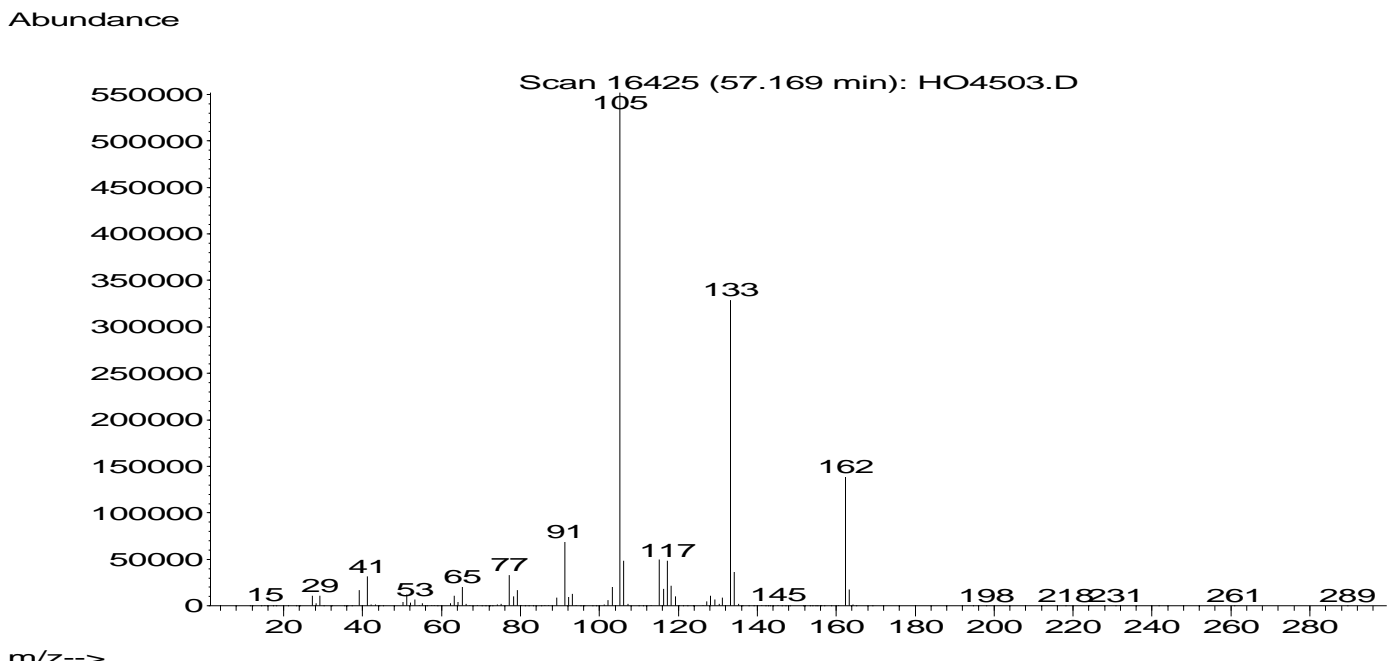

Figure S2c. Electron impact mass spectrum of (d) m-1-methylbutyltoluene.

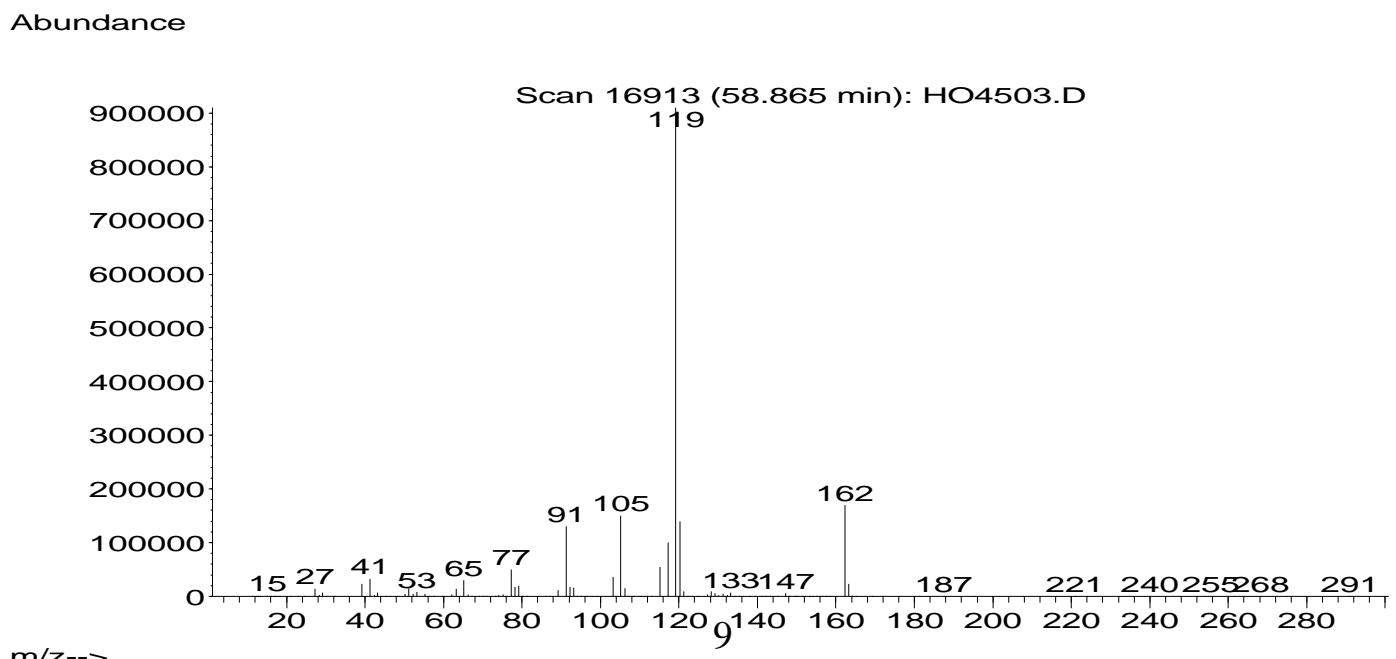


Figure S3. Proposed electron impact fragmentation sequence for 1-ethylpropyltoluene and 1methylbutyltoluene.
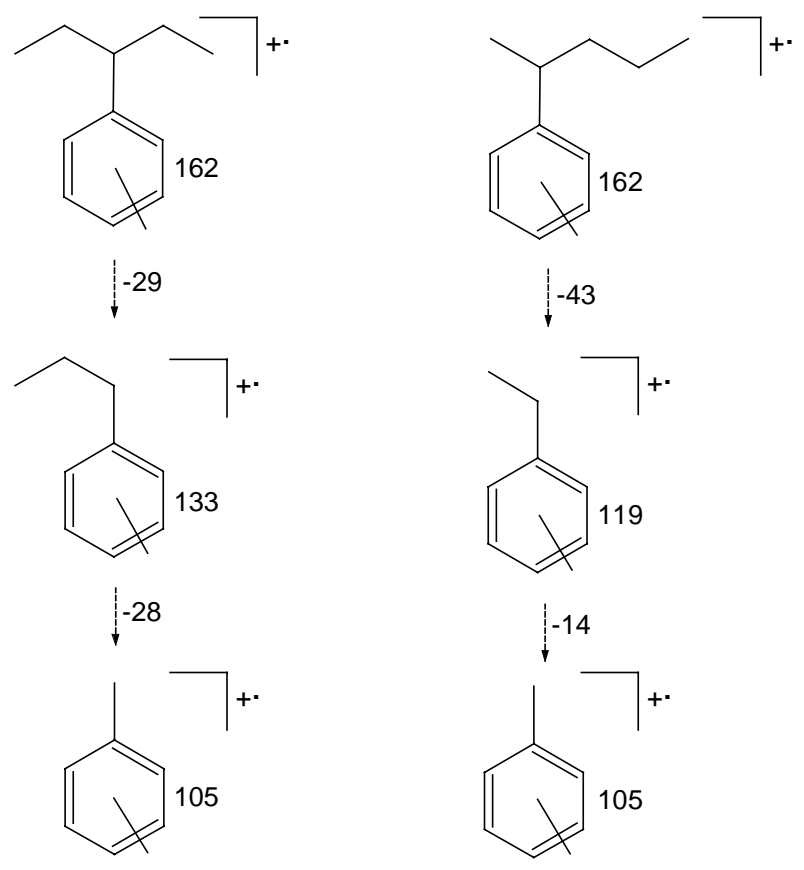
Figure S4a. Chromatogram of $\mathrm{AlCl}_{3}$ catalysed alkylation of toluene with 3-methyl-1-butene showing: (a) p-1,2-dimethylpropyltoluene, (b) p-1,1-dimethylpropyltoluene, (c) m-1,2dimethylpropyltoluene and (d) $m$-1,1-dimethylpropyltoluene.

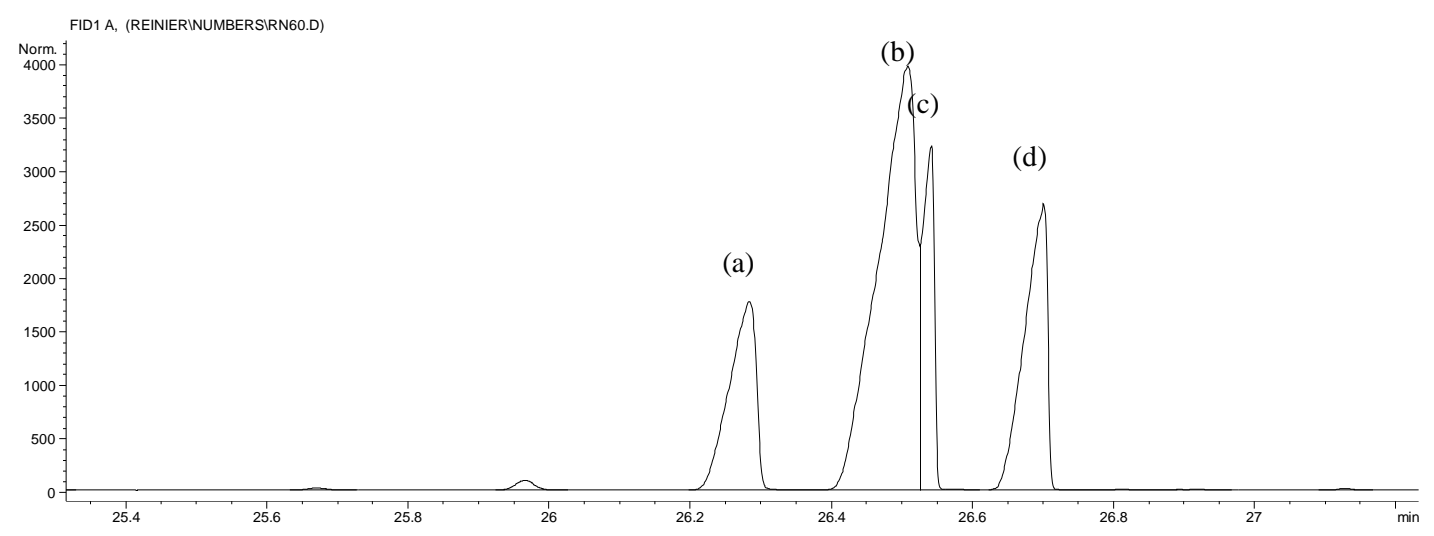

Figure S4b. Electron impact mass spectrum of (a) p-1,2-dimethylpropyltoluene.

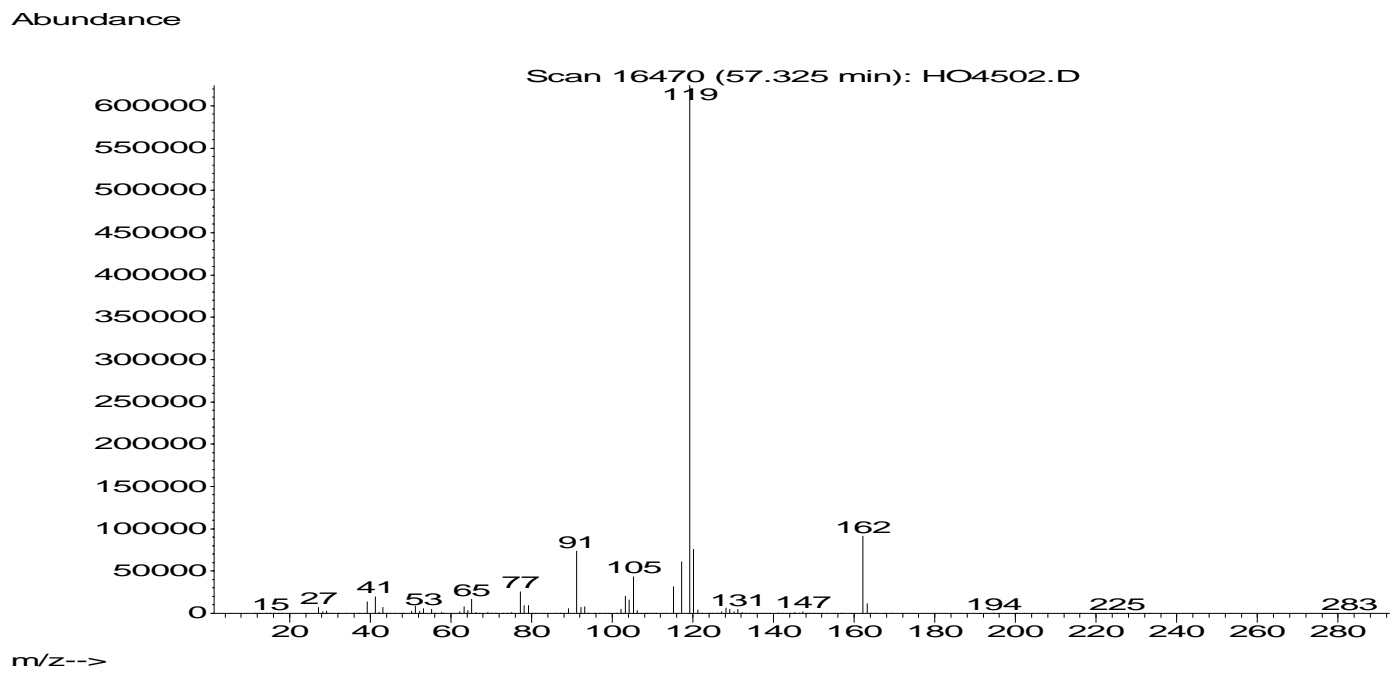

Figure S4c. Electron impact mass spectrum of (b) p-1,1-dimethylpropyltoluene.

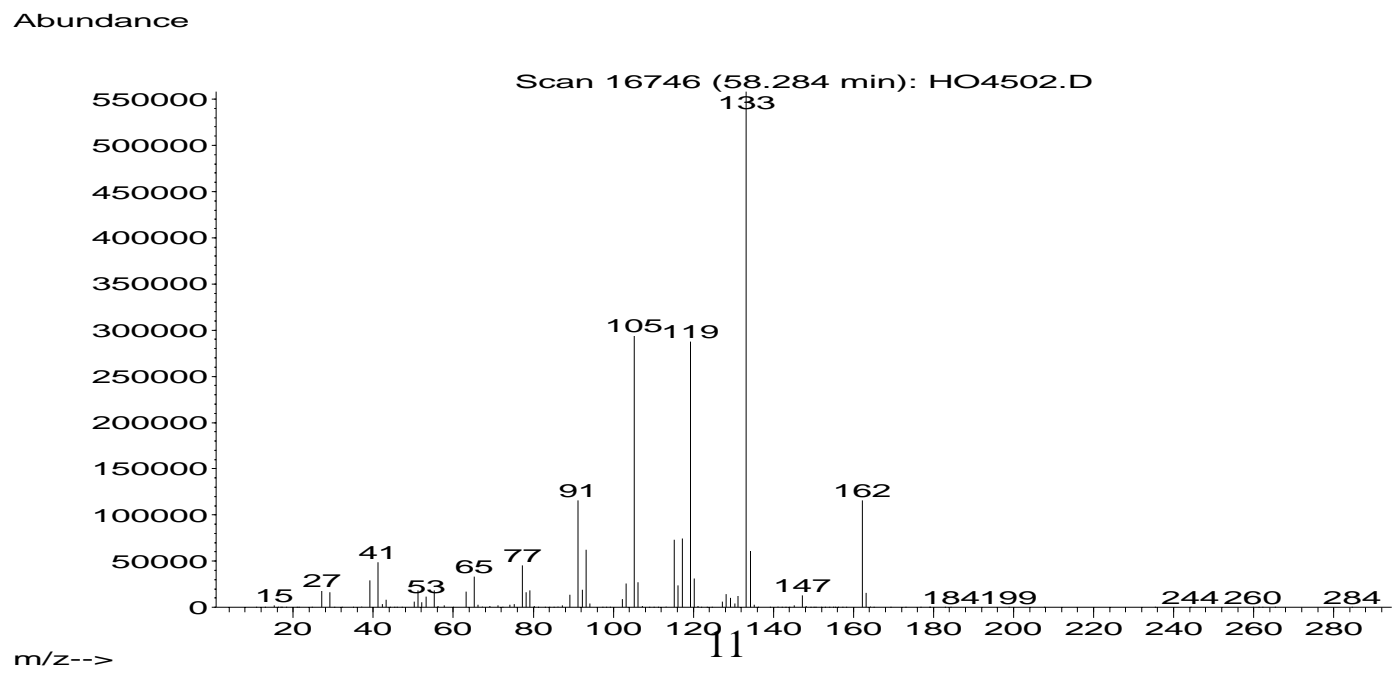


Figure S5. Proposed electron impact fragmentation sequence for 1,1-dimethylpropyltoluene and 1,2-dimethylpropyltoluene.
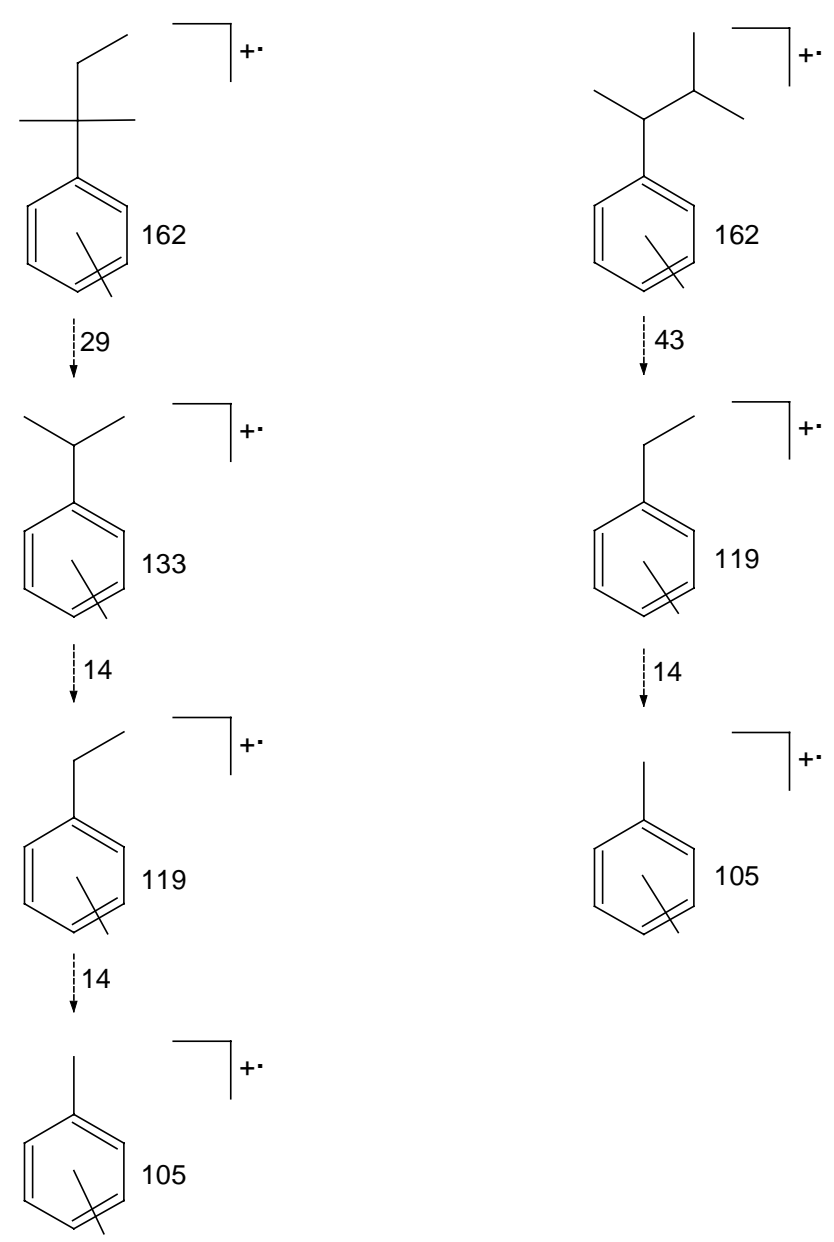
Figure S6. Chromatograms of diamyltoluene (--), di-isoamyltoluene (---) and decyltoluene (

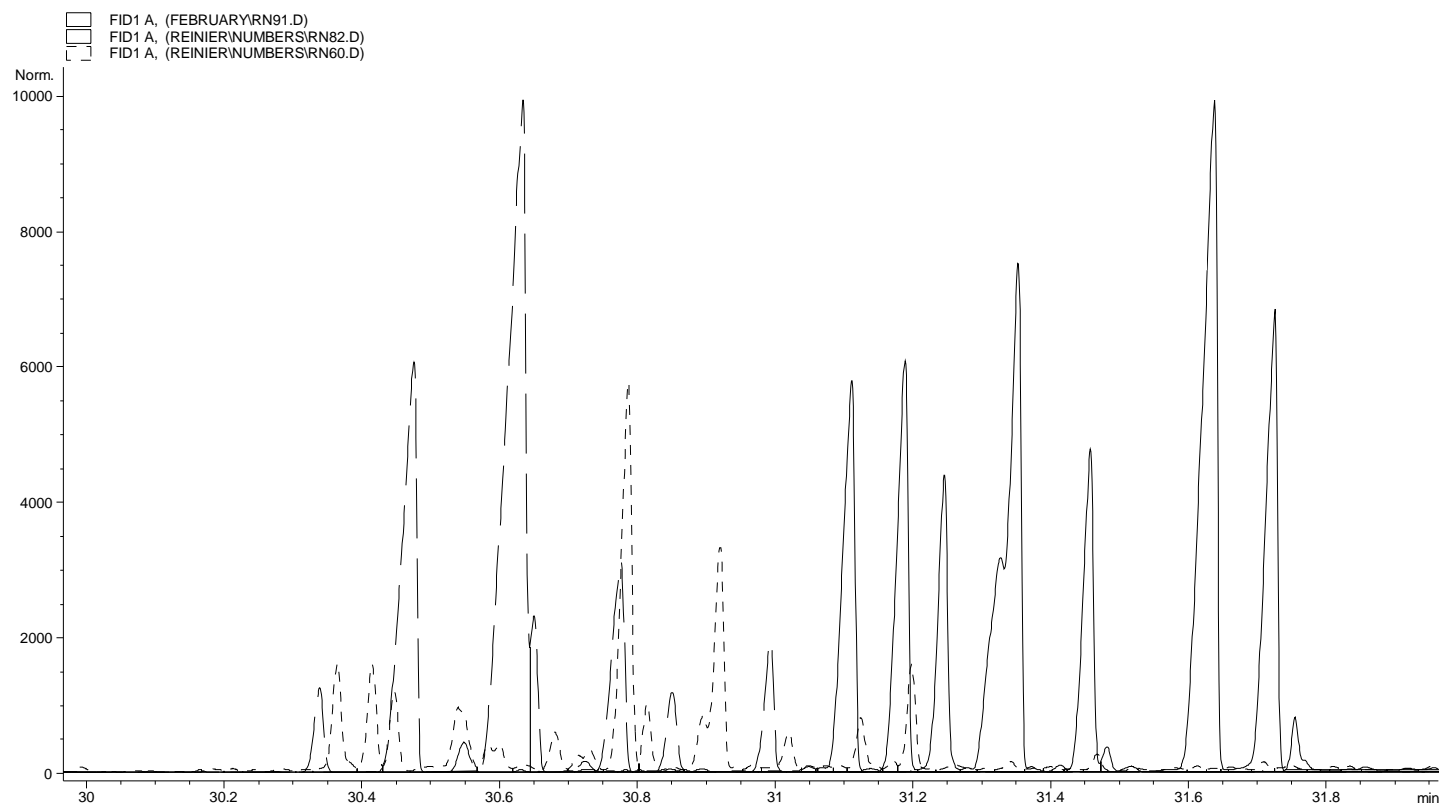

